\title{
Overexpression of membrane-type 2 matrix metalloproteinase induced by hypoxia-inducible factor-1 $\alpha$ in pancreatic cancer: Implications for tumor progression and prognosis
}

\author{
SHI-KAI ZHU ${ }^{1,3}$, YU ZHOU ${ }^{2,3}, \mathrm{CHAO} \mathrm{CHENG}^{4}$, SHAN ZHONG $^{1,3}, \mathrm{HAN}^{-Q I N G ~ W U}{ }^{4}$, \\ BO WANG ${ }^{4}$, PING FAN ${ }^{4}$, JIONG-XIN XIONG ${ }^{4}$, HONG-JI YANG ${ }^{1,3}$ and HE-SHUI WU ${ }^{4}$
}

${ }^{1}$ Organ Transplant Center, and ${ }^{2}$ Institute of Laboratory Medicine, Sichuan Academy of Medical Sciences and Sichuan Provincial People's Hospital, Chengdu, Sichuan 610072; ${ }^{3}$ School of Medicine, University of Electronic Science and Technology of China, Chengdu, Sichuan; ${ }^{4}$ Department of Pancreatic Surgery, Union Hospital, Tongji Medical College, Huazhong University of Science and Technology, Wuhan, Hubei 430022, P.R. China

Received May 20, 2014; Accepted July 15, 2014

DOI: $10.3892 / \mathrm{mco} .2014 .357$

\begin{abstract}
Membrane-type 2 matrix metalloproteinase (MT2-MMP) has been identified as a powerful modulator of the pericellular environment that promotes tumor invasion and metastasis. In this study, we investigated the association of MT2-MMP and hypoxia-inducible factor-1 $\alpha$ (HIF-1 $\alpha)$ expression in pancreatic cancer with regard to their clinical prognostic significance. Of the tissue specimens obtained from the 78 patients included in this study, $46(59 \%)$ were found to be positive for MT2-MMP immunostaining and MT2-MMP expression was colocalized with HIF-1 $\alpha$ in pancreatic cancer. Using the Spearman's rank analysis, the protein and mRNA expression level of MT2-MMP was found to be significantly correlated with HIF-1 $\alpha$ and CD34-microvascular density in pancreatic cancer. Furthermore, the expression of MT2-MMP in response to hypoxia was increased in a time-dependent manner and the promoter luciferase reporter revealed upregulation of MT2-MMP expression induced by HIF-1 $\alpha$ in pancreatic cancer cells. Moreover, the Cox regression model indicated that MT2-MMP was an independent prognostic factor in patients with pancreatic cancer. Our results demonstrated that the overexpression of MT2-MMP was induced by HIF-1 $\alpha$ in response to hypoxia and was an independent prognostic factor for pancreatic cancer progression.
\end{abstract}

Correspondence to: Dr Yu Zhou, Institute of Laboratory Medicine, Sichuan Academy of Medical Sciences and Sichuan Provincial People's Hospital, 32 West Second Section First Ring Road, Chengdu, Sichuan 610072, P.R. China

E-mail: zhushikai37@163.com

Key words: membrane-type 2 matrix metalloproteinase, pancreatic cancer, hypoxia-inducible factor-1 $\alpha$, tumor progression, prognosis

\section{Introduction}

Pancreatic cancer remains one of the most aggressive and intractable malignant tumors and is currently the fourth leading cause of cancer-related mortality worldwide (1). Due to its extremely high invasive and metastatic potential, pancreatic cancer is usually diagnosed at an advanced stage, leaving no adequate time window for effective systemic therapies and often recurs even following curative surgical excision (2). Despite significant advances in cancer therapy, including surgery, radiation, chemotherapy, or a combination of these approaches, the overall pancreatic cancer mortality rate has not been significantly reduced (3-5). Therefore, novel therapeutic approaches are required to improve the prognosis of pancreatic cancer patients.

A hypoxic tumor microenvironment is critical for tumor development, invasion and metastasis. Hypoxia-inducible factor-1 (HIF-1) plays a key role in cellular adaptation to hypoxia. Although HIF-1 is usually strongly suppressed by post-translational mechanisms under normoxic conditions, the activation of HIF-1 during hypoxia may upregulate hypoxia-induced gene expression, promote clonal selection of viable hypoxic tumor cells and drive the tumor toward a more aggressive phenotype, leading to invasion and metastasis $(6,7)$. Recent studies confirmed that elevated levels of hypoxia-induced gene expression have been found in pancreatic cancer cells, suggesting that HIF-1 $\alpha$ plays a crucial role in malignant tumor progression $(8,9)$. However, the molecular mechanisms through which the hypoxic microenvironment promotes pancreatic cancer invasion and metastasis have not been fully elucidated.

As is the case for the majority of solid malignant tumors, pancreatic cancer development is a multistep process, involving uncontrolled tumor growth, angiogenesis, detachment from the matrix, invasion and metastasis. Tumor invasion and metastasis have been shown to involve proteolytic degradation of the extracellular matrix, which is accomplished primarily by members of the matrix metalloproteinase (MMP) family. MMPs have been implicated in a number of physiological 
and pathological processes, including cellular migration, angiogenesis and invasion and metastasis of tumor cells (10). Indeed, MMPs are continuously overexpressed in a variety of malignant solid tumors and are involved in extracellular matrix destruction, thereby contributing to tumor invasion and metastasis $(11,12)$. Among those MMPs, membrane-type MMPs (MT-MMPs) constitute a specific subtype, conducting pericellular proteolysis, which is considered to be an important step in pericancerous tissue remodeling $(13,14)$.

MT-MMPs, which constitute a subfamily of six distinct membrane-associated MMPs, are $\mathrm{Zn}^{2+}$-binding endopeptidases that degrade various components of the extracellular matrix. MT2-MMP (also referred to as MMP-15), was the second member of the MT-MMP subfamily to be discovered, was originally isolated from a human lung cDNA library (15) and has been found to be constitutively expressed at low levels in normal tissues, but highly expressed in malignant tumors (16-19). MT2-MMP has been identified as a powerful modulator of the pericellular environment, promoting tumor growth, angiogenesis, invasion and metastasis (20). Moreover, MT2-MMP was also characterized as a new element in the anti-apoptotic pathway network of human tumor cells (21). Therefore, MT2-MMP has been considered to be particularly important for the malignant behavior of cancer cells.

Although we previously demonstrated that the upregulation of MT2-MMP in response to hypoxia is promoted by HIF-1 $\alpha$ in cancer cells (22), the available information on the association of the expression of MT2-MMP and HIF-1 $\alpha$ with clinical outcomes is limited. This study was undertaken to investigate the regional expression of MT2-MMP and HIF-1 $\alpha$ in pancreatic cancer, study the association of MT2-MMP and HIF-1 $\alpha$ expression with tumor progression and assess relevant clinicopathological factors for patient survival.

\section{Materials and methods}

Patients and surgical specimens. A total of 78 patients with pancreatic cancer who underwent pancreatectomy at the Department of Pancreatic Surgery, Union Hospital, Huazhong University of Science and Technology (HUST; Wuhan, China) between December, 2005 and September, 2010 were included in this retrospective study. The surgical specimens were collected, immediately placed in liquid nitrogen and stored at $-80^{\circ} \mathrm{C}$ until analysis. The diagnosis of all the cases was confirmed based on the World Health Organization classification, staged according to the tumor-node-metastasis (TNM) classification and were reviewed by two pathologists (23). Clinicopathological parameters, including age at surgery, gender, location of tumor, size of tumor, perineural invasion, vascular invasion, lymph node status, distant metastasis, TNM stage and tumor differentiation, were retrieved from patient records. The overall survival time was defined as the time from surgical resection to date of last follow-up or death. The analysis of human tissues was approved by the Human Research Ethics Committee of HUST and all the patients provided written informed consent on the use of their clinical specimens for medical research.

Immunohistochemistry (IHC). The expression of MT2-MMP, HIF-1 $\alpha$ and CD34 was determined using Histostain-Plus kits
(Zymed Laboratories, San Francisco, CA, USA), as previously described (24). In brief, serial tissue sections (4 $\mu \mathrm{m})$ from formalin-fixed and paraffin-embedded specimens were deparaffinized in xylene and rehydrated. All the sections were then immersed in $3 \%$ hydrogen peroxide for 30 min to block endogenous peroxidase activity. After neutralization of endogenous peroxidase, sections on glass slides were preincubated in blocking serum and were then incubated overnight at $4^{\circ} \mathrm{C}$ with anti-MT2-MMP antibody (Abcam, Inc., Cambridge, MA, USA), anti-HIF-1 $\alpha$ antibody (Santa Cruz Biotechnology, Inc., Santa Cruz, CA, USA) and monoclonal anti-CD34 antibody (Boster Biotechnology, Wuhan, China) at a 1:50 dilution. After three washes in phosphate-buffered saline (PBS), the sections were incubated for $2 \mathrm{~h}$ with biotinylated anti-mouse IgG, washed three times with PBS, incubated with avidin-biotin-peroxidase complex for $1 \mathrm{~h}$ and again washed for 10 min with PBS. The reaction products were visualized with 3,3'-diaminobenzidine tetrahydrochloride and the slides were counterstained with hematoxylin. A consecutive section from each specimen processed with normal mouse IgG (Boster Biotechnology) was used as a negative control.

The slides were evaluated twice at different times by two independent investigators who were blinded to the pathological characteristics. The staining intensity of MT2-MMP and HIF-1 $\alpha$ expression was classified semi-quantitatively by the percentage of the stained cells and the intensity of the staining into four grades as follows: absent (grade 1), weak (grade 2), moderate (grade 3 ) and strong (grade 4). Grade 3 and 4 specimens were considered to be overexpressing MT2-MMP and HIF-1 $\alpha$ and were recorded as positive results in the statistical analysis. To compare the regional expression of MT2-MMP and HIF- $1 \alpha$ in pancreatic cancer, their expression was evaluated in serial sections of selected cases exhibiting MT2-MMP and HIF-1 $\alpha$ expression.

To visualize the microvessels, sections stained with anti-CD34 antibody were examined under a light microscope (Olympus Corporation, Tokyo, Japan) and the hotspots containing a large number of microvessels were identified at a low magnification (x100). Five randomly selected hotspots were observed and the number of microvessels was counted manually (magnification, $\mathrm{x} 200$ ). The microvascular density (MVD) was calculated by counting CD34-positive vascular endothelial cells, using the method previously described (25) and expressed as number of vessels per $0.74 \mathrm{~mm}^{2}$ (magnification, $\mathrm{x} 200$ ).

Cell culture and hypoxic treatment. PANC-1, BxPC-3 and AsPC-1 pancreatic cancer cells were maintained in Dulbecco's modified Eagle's medium supplemented with $10 \%$ fetal calf serum, $100 \mathrm{mg} / \mathrm{ml}$ penicillin and $100 \mathrm{mg} / \mathrm{ml}$ streptomycin and cultured at $37^{\circ} \mathrm{C}$ in a $5 \% \mathrm{CO}_{2}$ incubator. For the stably transfected cells, $600 \mathrm{mg} / \mathrm{ml} \mathrm{G} 418$ (Sigma-Aldrich, St. Louis, MO, USA) was added into the medium. For hypoxic treatment, the cells were exposed to hypoxic conditions of $1 \% \mathrm{O}_{2}$, $5 \% \mathrm{CO}_{2}$ and $94 \% \mathrm{~N}_{2}$, which was created by a hypoxic chamber (Billups-Rothenberg, Inc., San Diego, CA, USA). The medium was switched to serum-free medium (Invitrogen Life Technologies, Carlsbad, CA, USA) before the cells were subjected to hypoxia and control cultures were grown in serum-free medium under normoxia in a $5 \% \mathrm{CO}_{2}$ incubator (Forma Scientific Co., Marietta, OH, USA). After incubation 
for the desired periods, the cells were harvested for subsequent experiments.

Reverse transcription-quantitative polymerase chain reaction (RT-qPCR). Total cellular RNA was isolated from each sample using the TRIzol reagent (Invitrogen Life Technologies) and subjected to DNase treatment. cDNA was prepared using the TaqMan Reverse Transcription Reagents kit (Applied Biosystems, Foster City, CA, USA) according to the manufacturer's instructions. The following reaction mixture was used for all the RT-qPCR samples: $1 X$ of iQ SYBR-Green supermix (Bio-Rad, Hercules, CA, USA), $200 \mathrm{nmol} / 1$ of each primer and $2.5 \mu \mathrm{l}$ of cDNA in a total volume of $25 \mu \mathrm{l}$. The reactions were amplified and analyzed using the ABI-7000 system (Applied Biosystems). The MT2-MMP primers were 5'-CTTCGG CTTTATGGCTACCT-3' (forward) and 5'-AAGGTCAGA TGGTGGTTGTTC-3' (reverse), whereas the primers used for HIF-1 $\alpha$ amplification were 5'-CATCTCCATCTCCTACCC ACA-3' (forward) and 5'-CTCAAAGCGACAGATAAC ACG-3' (reverse). $\beta$-actin was used as an internal control and the data were averaged from three individual experiments.

Western blotting. The cells were treated under normoxia or hypoxia and lysed by lysis buffer [50 mM Tris (pH 7.2), $1 \%$ Triton $\mathrm{X}-100,0.5 \%$ sodium deoxycholate, $0.1 \%$ sodium dodecyl sulphate (SDS), $500 \mathrm{mM} \mathrm{NaCl}, 10 \mathrm{mM} \mathrm{MgCl}_{2}$ with $1 \mathrm{mM}$ phenylmethanesulfonyl fluoride]. The proteins were separated by SDS-PAGE at $100 \mathrm{~V}$ for $1 \mathrm{~h}$ and transferred onto polyvinylidene difluoride membranes (Millipore, Billerica, MA, USA). Non-specific binding sites were blocked with $5 \%$ milk in Tris-buffered saline with Tween-20 [120 mM Tris- $\mathrm{HCl}$ (pH 7.4), $150 \mathrm{mM} \mathrm{NaCl}$ and $0.05 \%$ Tween-20] for $1 \mathrm{~h}$ at room temperature. The membranes were incubated with primary antibody against HIF-1 $\alpha$ or MT2-MMP overnight at $4^{\circ} \mathrm{C}$. The membranes were washed three times and incubated with horseradish peroxidase-conjugated secondary antibody and the proteins were visualized by ECL Western Blotting substrate (Pierce Biotechnology, Inc., Rockford, IL, USA). $\beta$-actin was used as an internal control and the data were averaged from three individual experiments.

Luciferase activity assay. A 956-bp MT2-MMP promoter construct, corresponding to the sequence from -855 to +101 (relative to the transcriptional start site) of the 5'-flanking region of the human MT2-MMP gene, was generated from human genomic DNA using F1 (5'-TTTGCTAGCAAG TGAGTCCGTGGAAATGATAG-3') and R1 (5'-TTTCTC GAGCGCCTGGGAAGAATGAAGAC-3') as forward and reverse primers, respectively. The cDNA of MT2-MMP was amplified by PCR and sequenced, then cloned into the pcDNA3.1/histidine (His) vector(Invitrogen Life Technologies) to yield a His-tagged MT2-MMP. The full-length HIF-1 $\alpha$ expression plasmid HA-HIF-1 $\alpha$-pcDNA3 was purchased from Addgene, Inc. (www.addgene.org; Cambridge MA, USA). Each promoter construct was co-transfected with the pRL-TK plasmid into subconfluent (80-90\%) monolayer cells using Lipofectamine 2000 (Invitrogen Life Technologies) according to the manufacturer's protocol. The transfection efficiency was determined as the percentage of control green fluorescent protein-expressing cells counted by flow cytometry. After
4-6 h of transfection, the cells were washed and allowed to recover overnight in fresh medium. Approximately $12 \mathrm{~h}$ later, the transfected cells were incubated under normoxia or hypoxia for $12 \mathrm{~h}$. Luciferase activity detection was performed using the Dual-Luciferase Reporter Assay system (Promega Corporation, Madison, WI, USA) according to the manufacturer's instructions.

Statistical analysis. All the statistical analyses were performed using the IBM SPSS Statistics version 20.0 (IBM Corp., New York, NY, USA). The significance of the difference between the incidence of MT2-MMP and HIF-1 $\alpha$ expression and several clinical and pathological parameters was assessed by the $\chi^{2}$ test or the Mann-Whitney t-test. The Pearson's correlation was used to evaluate the association between MT2-MMP and HIF-1 $\alpha$ by computing the correlation coefficient (r) and corresponding P-values. The Cox proportional hazards regression model was used for multivariate analyses. The Fisher's exact test was applied to assess the association between categorical variables. The overall survival time was calculated using the Kaplan-Meier method from the date of surgery to the date of death from pancreatic cancer and compared by the log-rank test. All the P-values were based on two-tailed statistical analysis and $\mathrm{P}<0.05$ was considered to indicate a statistically significant difference.

\section{Results}

Patients. All the patients were diagnosed with pancreatic cancer and none had received radiotherapy or chemotherapy prior to surgery. The demographic data and tumor characteristics are summarized in Table I. The patients included 45 men and 33 women, with a median age of 54 years (range, 23-78 years). At the time of last clinical follow-up (September, 2012), 15 patients $(19.2 \%)$ remained alive. The majority of the patients $(73.1 \%)$ had undergone radical surgery, whereas 21 patients $(27.9 \%)$ received palliative surgery. If indicated, the patients received standard gemcitabine-based chemotherapy, according to their clinical stage and performance status. No significant difference was noted between the MT2-MMP and HIF-1 $\alpha$ expression with respect to clinicopathological parameters such as age at surgery, gender, location of tumor, size of tumor, perineural invasion, vascular invasion, lymph node status, distant metastasis, TNM stage and differentiation $(\mathrm{P}>0.05$, Table I).

Expression of MT2-MMP and HIF-1 $\alpha$ in pancreatic cancer. The expression of the MT2-MMP and HIF-1 $\alpha$ proteins was assessed by IHC in tissue specimens from 78 patients with pancreatic cancer. The expression of the MT2-MMP protein was mainly localised to the plasma membrane and cytoplasm of tumor cells, whereas the nuclei of stromal cells (including fibroblasts and endothelial cells) were also occasionally stained. By contrast, the distribution of HIF-1 $\alpha$ expression was predominantly reflected by diffuse staining of the nucleus and/or cytoplasm of the tumor cells (Fig. 1). Of the 78 tissue specimens, 46 were positive $(59 \%)$ and 32 were negative (41\%) for MT2-MMP expression, whereas 45 tissue specimens $(58 \%)$ were positive for HIF-1 $\alpha$ expression. Of note, we observed that the expression of HIF-1 $\alpha$ and MT2-MMP overlapped when MT2-MMP 
Table I. Patient characteristics and frequency of MT2-MMP and HIF-1 $\alpha$ expression in pancreatic cancer.

\begin{tabular}{|c|c|c|c|c|c|c|c|}
\hline \multirow[b]{2}{*}{ Variables } & \multirow[b]{2}{*}{ Total, n (\%) } & \multicolumn{3}{|c|}{ MT2-MMP } & \multicolumn{3}{|c|}{ HIF-1 $\alpha$} \\
\hline & & Negative, n (\%) & Positive, n (\%) & P-value & Negative, $\mathrm{n}(\%)$ & Positive, n (\%) & P-value \\
\hline Age at surgery, years & & & & 0.598 & & & 0.237 \\
\hline$<60$ & $46(59)$ & $20(44)$ & $26(56)$ & & $22(48)$ & $24(52)$ & \\
\hline$\geq 60$ & $32(41)$ & $12(38)$ & $20(62)$ & & $11(34)$ & $21(66)$ & \\
\hline Gender & & & & 0.251 & & & 0.656 \\
\hline Male & $45(58)$ & $16(36)$ & $29(64)$ & & $20(44)$ & $25(56)$ & \\
\hline Female & $33(42)$ & $16(49)$ & $17(51)$ & & $13(40)$ & $20(60)$ & \\
\hline Tumor location & & & & 0.475 & & & 0.686 \\
\hline Head & $50(64)$ & $22(44)$ & $28(56)$ & & $22(44)$ & $28(56)$ & \\
\hline Body and tail & $28(36)$ & $10(36)$ & $18(64)$ & & $11(39)$ & $17(61)$ & \\
\hline Tumor size, $\mathrm{cm}$ & & & & 0.233 & & & 0.174 \\
\hline$<2$ & $50(64)$ & $23(46)$ & $27(54)$ & & $24(48)$ & $26(52)$ & \\
\hline$\geq 2$ & $28(36)$ & $9(32)$ & $19(68)$ & & $9(32)$ & $19(68)$ & \\
\hline Perineural invasion & & & & 0.475 & & & 0.174 \\
\hline Absent & $50(64)$ & $22(44)$ & $28(56)$ & & $24(48)$ & $26(52)$ & \\
\hline Present & $28(36)$ & $10(36)$ & $18(64)$ & & $9(32)$ & $19(68)$ & \\
\hline Vascular invasion & & & & 0.208 & & & 0.052 \\
\hline Absent & $63(81)$ & $28(44)$ & $35(56)$ & & $30(48)$ & $33(52)$ & \\
\hline Present & $15(19)$ & $4(27)$ & $11(74)$ & & $3(20)$ & $12(80)$ & \\
\hline Lymph node status & & & & 0.673 & & & 0.359 \\
\hline Absent & $54(69)$ & $23(43)$ & $31(57)$ & & $21(39)$ & $33(61)$ & \\
\hline Present & $24(31)$ & $9(38)$ & $15(62)$ & & $12(50)$ & $12(50)$ & \\
\hline Distant metastasis & & & & 0.944 & & & 0.399 \\
\hline Absent & $68(87)$ & $28(41)$ & $40(59)$ & & $30(44)$ & $38(56)$ & \\
\hline Present & $10(13)$ & $4(40)$ & $6(60)$ & & $3(30)$ & $7(70)$ & \\
\hline TNM stage & & & & 0.536 & & & 0.538 \\
\hline I+II & $48(62)$ & $21(44)$ & $27(56)$ & & $19(40)$ & $29(60)$ & \\
\hline III+IV & $30(38)$ & $11(37)$ & $19(63)$ & & $14(47)$ & $16(53)$ & \\
\hline Cell differentiation & & & & 0.251 & & & 0.061 \\
\hline $\mathrm{I}+\mathrm{II}$ & $33(42)$ & $16(48)$ & $17(52)$ & & $18(54)$ & $15(46)$ & \\
\hline III+IV & $45(58)$ & $16(36)$ & $29(64)$ & & $15(33)$ & $30(67)$ & \\
\hline Total no. of patients & $78(100)$ & $32(41)$ & $46(59)$ & & $33(42)$ & $45(58)$ & \\
\hline
\end{tabular}

MT2-MMP, membrane type-2 matrix metalloproteinase; HIF-1 $\alpha$, hypoxia-inducible factor-1 $\alpha$.

expression was compared to HIF-1 $\alpha$ expression in consecutive sections of the same tumor, suggesting a spatially coincident expression (Fig. 1). In addition, the positive expression of CD34 was mainly presented as brownish or brownish-yellow granules in the cytoplasm of microvascular endothelial cells.

Correlation between MT2-MMP, HIF-1 $\alpha$ expression and CD34-MVD in pancreatic cancer. Using the Spearman's rank correlation coefficient, the immunostaining of MT2-MMP was confirmed to be significantly correlated with that of HIF-1 $\alpha$ in pancreatic cancer tissues $(r=0.516, \mathrm{P}<0.001$; Fig. 2A). Furthermore, we confirmed that the expression of HIF-1 $\alpha$ mRNA was also correlated with that of MT2-MMP ( $r=0.656$, $\mathrm{P}<0.001$; Fig. 2B). The CD34-MVD in the MT2-MMP-positive tumors was $40.7 \pm 15.4 / 0.74 \mathrm{~mm}^{2}$, which was significantly higher compared to that in MT2-MMP-negative tumors $\left(24.2 \pm 10.8 / 0.74 \mathrm{~mm}^{2} ; \mathrm{P}<0.001\right)$. The CD34-MVD in HIF-1 $\alpha$-positive tumors was $39.4 \pm 14.9 / 0.74 \mathrm{~mm}^{2}$, which was significantly higher compared to that in HIF-1 $\alpha$-negative tumors $\left(26.4 \pm 14.2 / 0.74 \mathrm{~mm}^{2} ; \mathrm{P}<0.001\right)$. Furthermore, we also observed that CD34-MVD was significantly associated with the expression of MT2-MMP ( $\mathrm{r}=0.686, \mathrm{P}<0.001$; Fig. $2 \mathrm{C}$ ) and HIF-1 $\alpha$ ( $r=0.867, \mathrm{P}<0.001$; Fig. 2D) in pancreatic cancer.

Hypoxia induces MT2-MMP expression in pancreatic cancer cells. Based on the correlation of MT2-MMP and HIF-1 $\alpha$ expression in pancreatic cancer tissues, we investigated the expression of MT2-MMP in response to hypoxia in three 
A

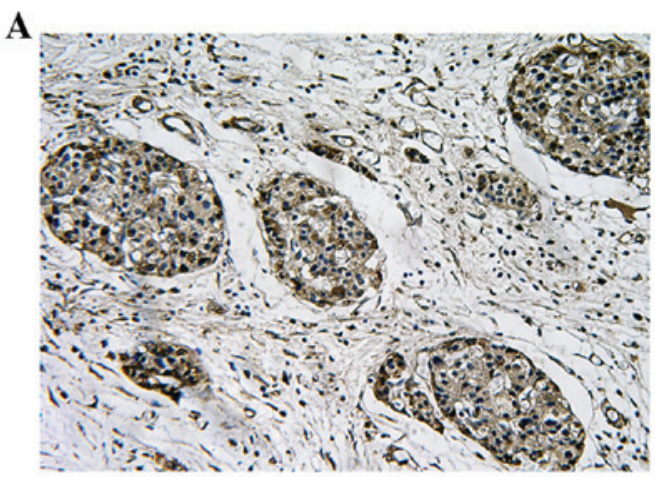

c
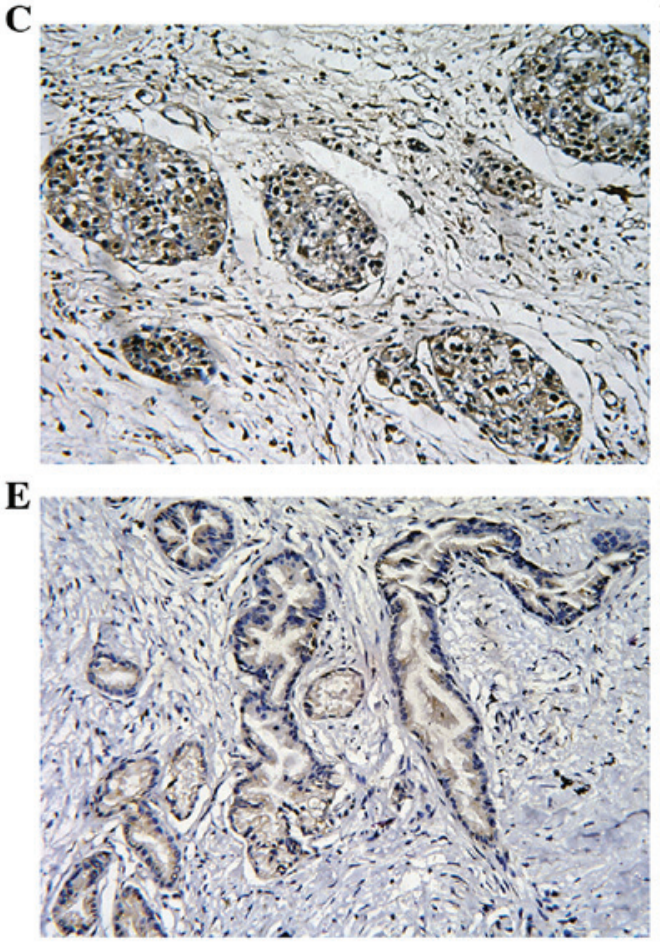

B

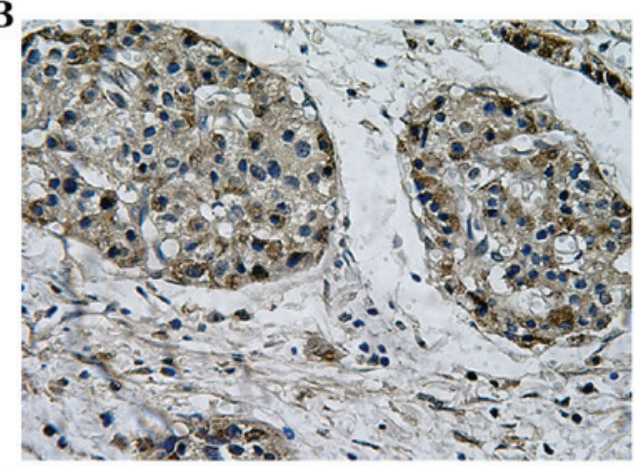

D

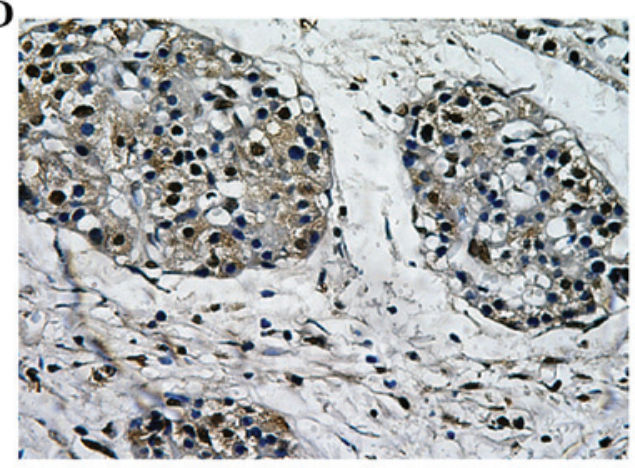

$\mathbf{F}$

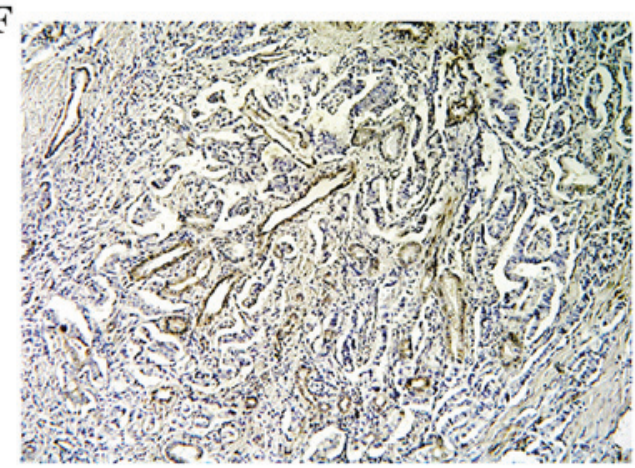

Figure 1. Representative images of membrane type-2 matrix metalloproteinase (MT2-MMP), hypoxia-inducible factor-1 $\alpha$ (HIF-1 $\alpha$ ) and CD34 expression in pancreatic cancer. (A and B) Positive immunostaining for MT2-MMP in pancreatic cancer tissues (A: magnification, x200; and B: magnification, x400). (C and D) Positive immunostaining for HIF-1 $\alpha$ in pancreatic cancer tissues (C: magnification, $x 200$; and D: magnification, $x 400$ ). (E) Negative immunostaining for MT2-MMP in pancreatic cancer tissues (magnification, $\mathrm{x} 200$ ). (F) Intensive immunostaining for microvessels with anti-CD34 staining in pancreatic cancer tissues (magnification, x200).

pancreatic cancer cell lines, namely PANC-1, BxPC-3 and AsPC-1. The levels of MT2-MMP expression were determined using RT-qPCR and western blotting when the cells were exposed to hypoxia $\left(1 \% \mathrm{O}_{2}\right)$ for 0,12 and $24 \mathrm{~h}$. The MT2-MMP expression at the mRNA and protein levels was markedly increased in a time-dependent manner following exposure to hypoxia for $12 \mathrm{~h}$ (Fig. 3). Moreover, the increased MT2-MMP expression was accompanied by a markedly increased HIF-1 $\alpha$ expression. Furthermore, the MT2-MMP promoter luciferase reporter was cotransfected with pRL-TK plasmids into the indicated cells and exposed to either normoxia or hypoxia for $12 \mathrm{~h}$; the data indicated that hypoxia induces MT2-MMP expression in pancreatic cancer cells.

Correlation between MT2-MMP, HIF-1 $\alpha$ expression and postoperative prognosis. Upon univariate analysis with the Cox proportional hazards model, MT2-MMP-positive patients exhibited a significantly poorer survival compared to
MT2-MMP-negative patients ( $\mathrm{P}<0.001$, Table II). The overall survival rate of MT2-MMP-positive and MT2-MMP-negative patients was found to be 4 and $41 \%$, respectively, and TNM stage was positively correlated with a poor prognosis $(\mathrm{P}=0.026)$. Furthermore, the multivariate analysis using the Cox regression model indicated that MT2-MMP expression was an independent predictor of an unfavorable prognosis $[\mathrm{P}=0.005$; hazard ratio $(\mathrm{HR})=2.445$; 95\% confidence interval $(\mathrm{CI})$ : 1.316-4.544], as were the presence of vascular invasion $(\mathrm{P}=0.012 ; \mathrm{HR}=3.427$; and 95\% CI: 1.622-7.241), TNM stage ( $\mathrm{P}=0.021 ; \mathrm{HR}=1.862$; and 95\% CI: 1.098-3.156), cell differentiation ( $\mathrm{P}=0.009$; $\mathrm{HR}=2.082$; and 95\% CI: $1.200-3.610)$ and HIF-1 $\alpha$ expression $(\mathrm{P}=0.001$; $\mathrm{HR}=3.120$; and 95\% CI: 1.600-6.082) (Table III). Moreover, the Kaplan-Meier survival curves demonstrated that the positive expression of the MT2-MMP protein was associated with a shorter disease-free survival $(\log -\mathrm{rank}=22.369, \mathrm{P}<0.001$, Fig. 4A), as was the positive expression of the HIF-1 $\alpha$ protein $(\log -$ rank $=33.017, \mathrm{P}<0.001$, Fig. 4B $)$. 

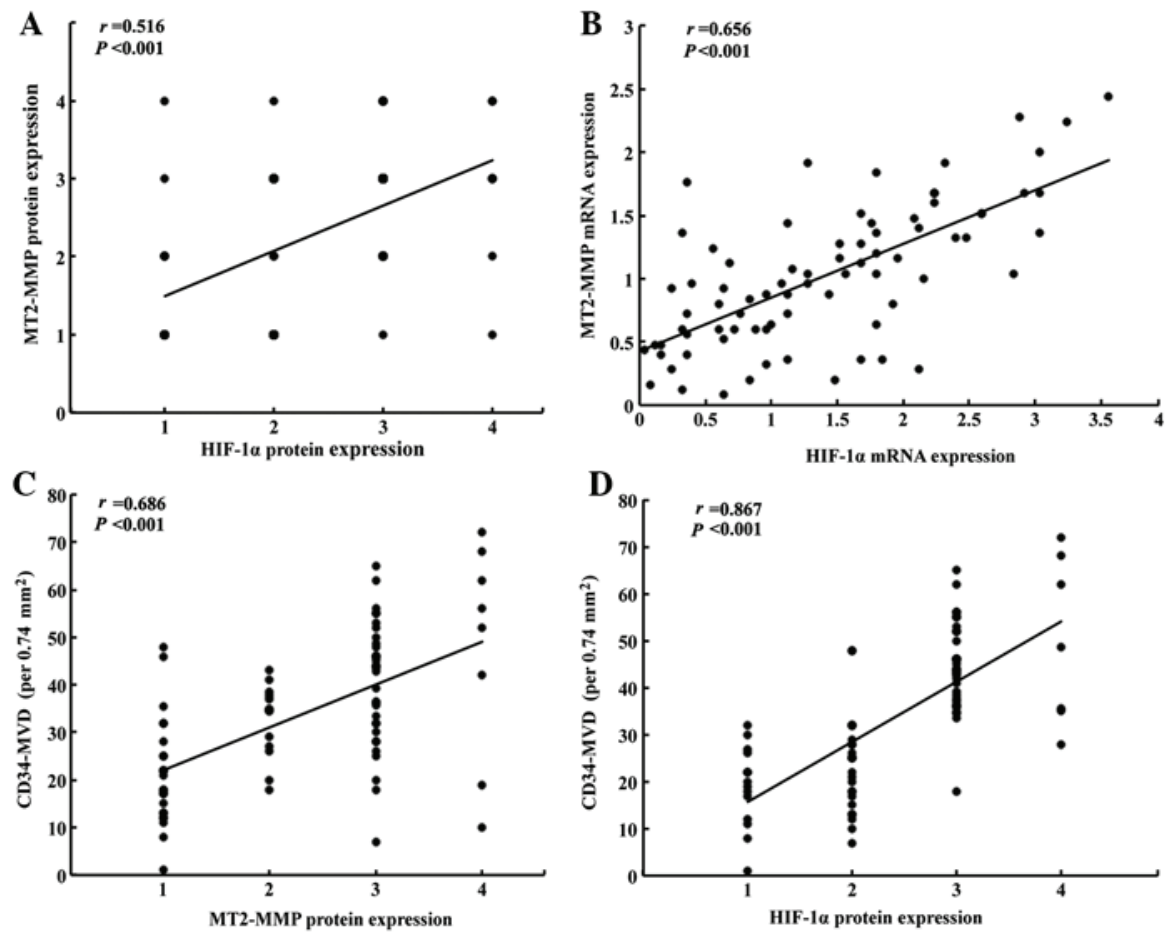

Figure 2. Correlations of membrane type-2 matrix metalloproteinase (MT2-MMP), hypoxia-inducible factor-1 $\alpha$ (HIF-1 $\alpha$ ) expression and CD34-microvascular density (MVD) in pancreatic cancer. (A) Scatter plot of HIF-1 $\alpha$ protein expression (grades 1-4) in pancreatic cancer with protein levels of MT2-MMP (grades 1-4) $(\mathrm{r}=0.516, \mathrm{P}<0.001)$. (B) Correlation between the expression of HIF-1 $\alpha$ and MT2-MMP mRNA in pancreatic cancer $(\mathrm{r}=0.656, \mathrm{P}<0.001)$ (C) Correlation between CD34-MVD and MT2-MMP protein expression (grades 1-4) in pancreatic cancer ( $r=0.686$, $P<0.001)$. (D) Correlation between CD34-MVD and HIF-1 $\alpha$ protein expression (grades 1-4) in pancreatic cancer $(\mathrm{r}=0.867, \mathrm{P}<0.001)$.

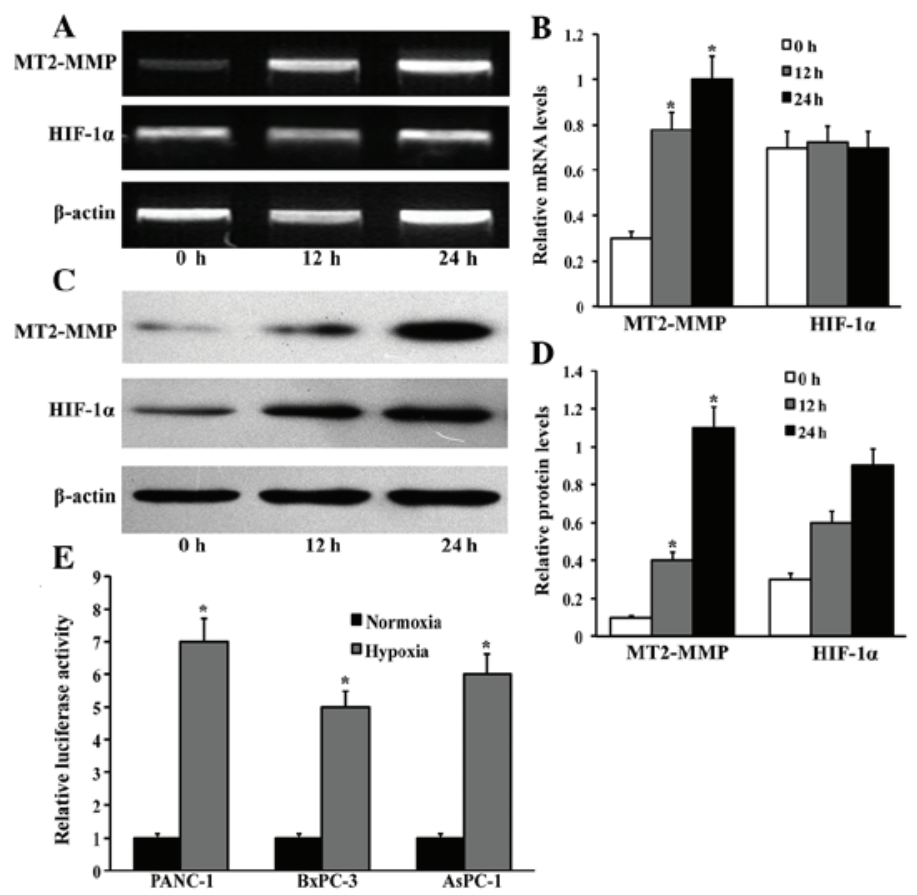

Figure 3. Hypoxia induces membrane type-2 matrix metalloproteinase (MT2-MMP) expression in pancreatic cancer. (A) Pancreatic cancer cells were exposed to hypoxia $\left(1 \% \mathrm{O}_{2}\right)$ at different time-points and the relative mRNA levels of MT2-MMP and hypoxia-inducible factor- $1 \alpha(\mathrm{HIF}-1 \alpha)$ expression were determined by quantitative polymerase chain reaction. (B) The densitometric analysis of MT2-MMP and HIF-1 $\alpha$ mRNA expression was performed by BandScan 5.0 software (Glyko Inc., Novato, CA, USA). The results are presented as the relative mRNA levels of MT2-MMP and HIF-1 $\alpha$ to $\beta$-actin. Columns, duplicate determinations in one representative experiment of three; bars, standard deviation (SD). "P<0.05, compared to the prior timepoints. (C) Protein levels of MT2-MMP and HIF-1 $\alpha$ after exposure to hypoxia $\left(1 \% \mathrm{O}_{2}\right)$ at different time-points were analyzed by western blotting in pancreatic cancer cells. (D) The densitometric analysis was preformed and the results were presented as the relative protein levels of MT2-MMP and HIF-1 $\alpha$ to $\beta$-actin. Columns, duplicate determinations in one representative experiment of three; bars, SD. "P $<0.05$, compared to the prior timepoints. (E) The MT2-MMP promoter luciferase reporter was cotransfected with pRL-TK plasmids into the indicated cells and exposed to either normoxia or hypoxia for $12 \mathrm{~h}$; the cells were then extracted and luciferase activity was detected. The results were expressed as means $\pm \mathrm{SD}$ and represent three independent experiments. ${ }^{*}<0.05$, compared to control. 
Table II. Correlation of MT2-MMP and HIF-1 $\alpha$ expression with overall survival of patients with pancreatic cancer.

\begin{tabular}{|c|c|c|c|c|c|}
\hline \multirow[b]{2}{*}{ Variables } & \multirow[b]{2}{*}{$\begin{array}{l}\text { Total, no. } \\
(\mathrm{n}=78)\end{array}$} & \multicolumn{2}{|c|}{ Status, no. (\%) } & \multirow[b]{2}{*}{$\begin{array}{c}\text { Overall } \\
\text { survival rate }(\%)\end{array}$} & \multirow[b]{2}{*}{ P-value } \\
\hline & & $\begin{array}{l}\text { Alive } \\
(n=15)\end{array}$ & $\begin{array}{c}\text { Deceased } \\
(n=63)\end{array}$ & & \\
\hline Age at surgery, years & & & & & 0.621 \\
\hline$<60$ & 46 & $8(17)$ & $38(83)$ & 17 & \\
\hline$\geq 60$ & 32 & $7(22)$ & $25(78)$ & 22 & \\
\hline Gender & & & & & 0.052 \\
\hline Male & 45 & $12(27)$ & $33(73)$ & 27 & \\
\hline Female & 33 & $3(9)$ & $30(91)$ & 9 & \\
\hline Tumor location & & & & & 0.407 \\
\hline Head & 50 & $11(22)$ & $39(78)$ & 22 & \\
\hline Body and tail & 28 & $4(14)$ & $24(86)$ & 14 & \\
\hline Tumor size, $\mathrm{cm}$ & & & & & 0.153 \\
\hline$<2$ & 50 & $12(24)$ & $38(76)$ & 24 & \\
\hline$\geq 2$ & 28 & $3(11)$ & $25(89)$ & 11 & \\
\hline Perineural invasion & & & & & 0.407 \\
\hline Absent & 50 & $11(22)$ & $39(78)$ & 22 & \\
\hline Present & 28 & $4(14)$ & $24(86)$ & 14 & \\
\hline Vascular invasion & & & & & 0.933 \\
\hline Absent & 63 & $12(19)$ & $51(81)$ & 19 & \\
\hline Present & 15 & $3(20)$ & $12(80)$ & 20 & \\
\hline Lymph node status & & & & & 0.104 \\
\hline Absent & 54 & $13(24)$ & $41(76)$ & 24 & \\
\hline Present & 24 & $2(8)$ & $22(92)$ & 8 & \\
\hline Distant metastasis & & & & & 0.947 \\
\hline Absent & 68 & $13(19)$ & $55(81)$ & 19 & \\
\hline Present & 10 & $2(20)$ & $8(80)$ & 20 & \\
\hline TNM stage & & & & & 0.026 \\
\hline $\mathrm{I}+\mathrm{II}$ & 48 & $13(27)$ & $35(73)$ & 27 & \\
\hline III+IV & 30 & $2(7)$ & $28(93)$ & 7 & \\
\hline Cell differentiation & & & & & 0.336 \\
\hline $\mathrm{I}+\mathrm{II}$ & 33 & $8(24)$ & $25(76)$ & 24 & \\
\hline $\mathrm{III}+\mathrm{IV}$ & 45 & $7(16)$ & $38(84)$ & 16 & \\
\hline MT2-MMP expression & & & & & $<0.001$ \\
\hline Negative & 32 & $13(41)$ & $19(59)$ & 41 & \\
\hline Positive & 46 & $2(4)$ & $44(96)$ & 4 & \\
\hline HIF-1 $\alpha$ expression & & & & & $<0.001$ \\
\hline Negative & 33 & $13(39)$ & $20(61)$ & 39 & \\
\hline Positive & 45 & $2(4)$ & $43(96)$ & 4 & \\
\hline
\end{tabular}

MT2-MMP, membrane type-2 matrix metalloproteinase; HIF-1 $\alpha$, hypoxia-inducible factor-1 $\alpha$.

\section{Discussion}

Tumor progression requires increased adaptation to a hypoxic microenvironment. Tumor hypoxia is an important characteristic of advanced pancreatic cancer, with a negative effect on patient prognosis. The evaluation of cellular responses to hypoxia may be of clinical relevance in a prognostic and predictive way and may also help treatment adaptation (26).
The transcription factor HIF-1 plays a central role in mediating this process. Inhibition of HIF-1 expression may lead to prevention of cell proliferation and growth, induction of cell apoptosis and arrest of tumor progression (27). MT2-MMP is known to play an important role in angiogenesis, tumor invasion and metastasis through the breakdown of the extracellular matrix (20). Although our previous study in vitro demonstrated the induction of MT2-MMP by HIF-1 $\alpha$ in cancer cells under 
Table III. Multivariate Cox analysis of overall survival of 78 patients with pancreatic cancer.

\begin{tabular}{lcccc}
\hline & & \multicolumn{3}{c}{ Multivariate analysis } \\
\cline { 3 - 4 } Variables & Univariate analysis & P-value & Hazard ratio & $95 \%$ CI \\
\hline Age at surgery, years & 0.621 & 0.149 & - & - \\
Gender & 0.052 & 0.598 & - & - \\
Tumor location & 0.407 & 0.299 & - & - \\
Tumor size, cm & 0.153 & 0.230 & - & - \\
Perineural invasion & 0.407 & 0.453 & - & $-622-7.241$ \\
Vascular invasion & 0.933 & 0.012 & 3.427 & - \\
Lymph node status & 0.104 & 0.959 & - & $1.098-3.156$ \\
Distant metastasis & 0.947 & 0.429 & - & $1.200-3.610$ \\
TNM stage & 0.026 & 0.021 & 1.862 & $1.316-4.544$ \\
Cell differentiation & 0.336 & 0.009 & 2.082 & $1.600-6.082$ \\
MT2-MMP & $<0.001$ & 0.005 & 2.445 & 3.120 \\
HIF-1 $\alpha$ & $<0.001$ & 0.001 & & \\
\hline
\end{tabular}

CI, confidence interval; MT2-MMP, membrane type-2 matrix metalloproteinase; HIF-1 $\alpha$, hypoxia-inducible factor- $1 \alpha$.
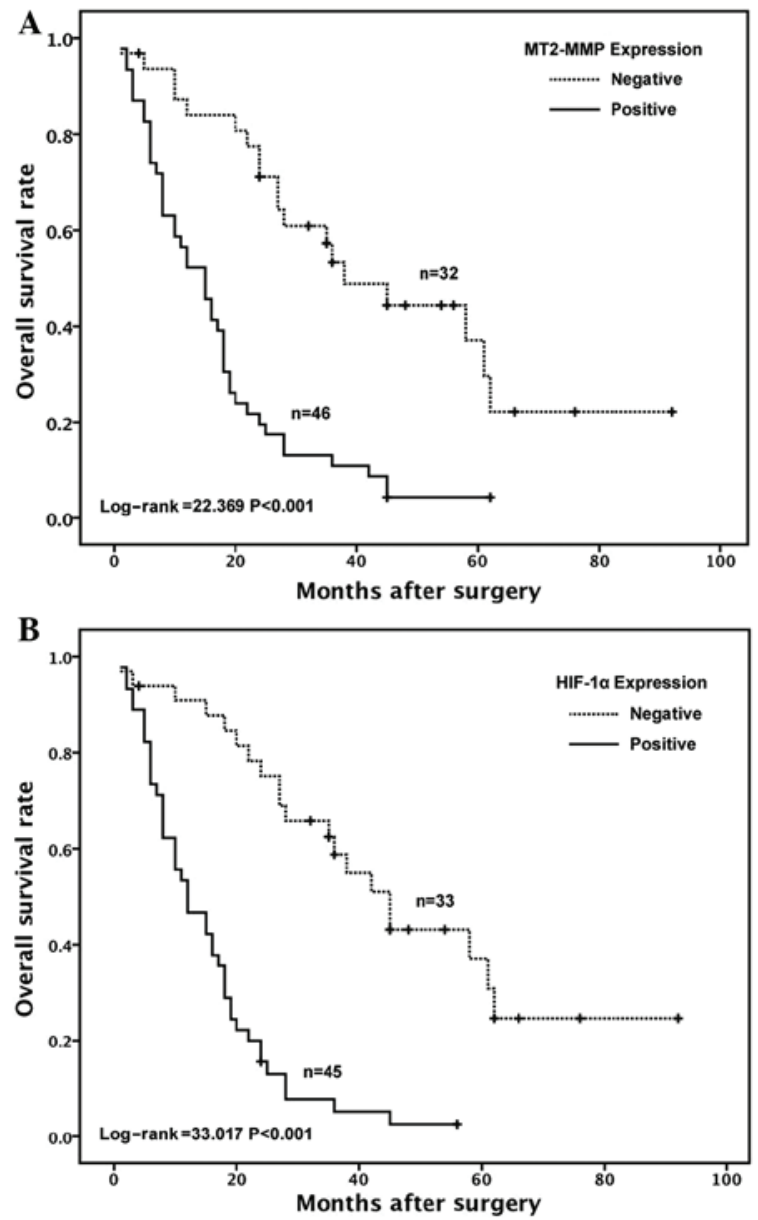

Figure 4. Kaplan-Meier survival curves according to membrane type-2 matrix metalloproteinase (MT2-MMP) and hypoxia-inducible factor-1 $\alpha$ (HIF-1 $\alpha)$ expression in pancreatic cancer. (A) Among all 78 cases, the percentage of MT2-MMP-positive cells was found to be associated with a shorter disease-free survival $(\mathrm{P}<0.001)$. (B) A high percentage of HIF-1 $\alpha$-positive cells was found to be associated with a shorter disease-free survival $(\mathrm{P}<0.001)$ The curves were constructed using the Kaplan-Meier method and compared using the log-rank test. The tick marks indicate censored data. hypoxia (22), to the best of our knowledge, the association of MT2-MMP expression and HIF-1 $\alpha$ has not yet been assessed in pancreatic cancer.

In the present study, a significant association between MT2-MMP and HIF-1 $\alpha$ expression was reported in an IHC study of pancreatic cancer. The expression of MT2-MMP was diffuse in pancreatic cancer tissues, unlike that of HIF-1 $\alpha$, which was focal and heterogeneous. When MT2-MMP expression was compared to that of HIF-1 $\alpha$ in consecutive sections of same tumor, almost all areas exhibiting MT2-MMP staining also exhibited HIF-1 $\alpha$ staining, although HIF-1 $\alpha$ staining was found in certain areas without MT2-MMP staining. Furthermore, we observed that MT2-MMP expression was markedly increased at the mRNA as well as the protein level, accompanied by increased levels of HIF-1 $\alpha$ expression when subjected to hypoxia. Those results suggested that HIF-1 $\alpha$ may play a regulatory role in the expression of MT2-MMP in pancreatic cancer.

HIF-1 $\alpha$ binds to hypoxia response elements in the promoter regions of HIF-1-targeted genes, thereby activating a large number of downstream genes involved in cell proliferation, differentiation, apoptosis, energy metabolism, tumor invasion and metastasis. In the present study, the MT2-MMP promoter luciferase reporter indicated that hypoxia induces MT2-MMP expression in pancreatic cancer cells though direct binding of HIF-1 $\alpha$ to the MT2-MMP promoter. Herein, these findings support the regulation of MT2-MMP expression by HIF-1 $\alpha$ in pancreatic cancer.

It is well recognized that angiogenesis is essential for the growth and progression of pancreatic cancer. MT2-MMP plays a vital role in the turnover of the basement membrane and exerts a significant effect on angiogenesis $(20,28,29)$. The CD34-MVD is a reliable index of tumor angiogenesis and reflects the potentiality of pancreatic cancer progression and prognosis $(30,31)$. In this study, we found that patients with a high level of CD34-MVD exhibited an inferior survival duration; furthermore, CD34-MVD was significantly 
associated with MT2-MMP expression in pancreatic cancer. Interestingly, a positive correlation was identified between MT2-MMP and HIF-1 $\alpha$ expression. Therefore, MT2-MMP expression induced by hypoxia appears to play a critical role in tumor development by enhancing tumor aggressiveness and malignant potential through the induction of processes such as neovascularization.

Considering the superior prognostic significance of MT2-MMP in the multivariate analysis, MT2-MMP expression status may be a more reliable marker for predicting tumor aggressiveness associated with tumor hypoxia. A growing body of evidence specifically implicates MT2-MMP in pancreatic cancer characterized by local invasion and a propensity to metastasize (32). MT2-MMP-positive patients exhibited a significantly poorer survival compared to MT2-MMP-negative patients.

The key findings of this study are that the overexpression of MT2-MMP is induced by hypoxia marker HIF-1 $\alpha$ in pancreatic cancer and that MT2-MMP overexpression is an independent predictor of poor clinical outcome and decreased survival. Therefore, the hypoxia-related marker MT2-MMP may be of significant value as a prognostic and predictive marker and as a potential therapeutic target in pancreatic cancer.

\section{Acknowledgements}

This study was supported by grants from the Scientific Research Fund of Sichuan Provincial Health Department, China (nos. 110207 and 130138) and the Young Doctor Fund of Sichuan Academy of Medical Sciences and Sichuan Provincial People's Hospital, China (no. 30305030562).

\section{References}

1. Siegel R, Naishadham D and Jemal A: Cancer statistics, 2013 CA Cancer J Clin 63: 11-30, 2013.

2. Traverso LW: Pancreatic cancer: surgery alone is not sufficient Surg Endosc 20 (Suppl 2): S446-S449, 2006

3. Frigeri M, De Dosso S, Castillo-Fernandez O, Feuerlein K, Neuenschwander H and Saletti P: Chemotherapy in patients with advanced pancreatic cancer: too close to death? Support Care Cancer 21: 157-163, 2013.

4. Vladov N, Takorov I, Kazarov K, et al: Surgical potentialities for the treatment of pancreatic cancer. Hepatogastroenterology 59: $280-283,2012$

5. Sultana A, Cox T, Ghaneh P and Neoptolemos JP: Adjuvant therapy for pancreatic cancer. Recent Results Cancer Res 196: 65-88, 2012.

6. Cavadas MA, Nguyen LK and Cheong A: Hypoxia-inducible factor (HIF) network: insights from mathematical models. Cell Commun Signal 11: 42, 2013.

7. Evans CE, Branco-Price C and Johnson RS: HIF-mediated endothelial response during cancer progression. Int J Hematol 95: 471-477, 2012

8. Shibaji T, Nagao M, Ikeda N, et al: Prognostic significance of HIF-1 alpha overexpression in human pancreatic cancer. Anticancer Res 23: 4721-4727, 2003.

9. Cheng ZX, Sun B, Wang SJ, et al: Nuclear factor-кB-dependent epithelial to mesenchymal transition induced by HIF-1 $\alpha$ activation in pancreatic cancer cells under hypoxic conditions. PLoS One 6: e23752, 2011.

10. Malemud CJ: Matrix metalloproteinases (MMPs) in health and disease: an overview. Front Biosci 11: 1696-1701, 2006.

11. Sato $\mathrm{H}$ and Takino T: Coordinate action of membrane-type matrix metalloproteinase-1 (MT1-MMP) and MMP-2 enhances pericellular proteolysis and invasion. Cancer Sci 101: 843-847, 2010.
12. Zhou C and Petroll WM: MMP regulation of corneal keratocyte motility and mechanics in 3-D collagen matrices. Exp Eye Res 121: 147-160, 2014.

13. Li J, Zucker S, Pulkoski-Gross A, et al: Conversion of stationary to invasive tumor initiating cells (TICs): role of hypoxia in membrane type 1-matrix metalloproteinase (MT1-MMP) trafficking. PLoS One 7: e38403, 2012.

14. Shaverdashvili K, Wong P, Ma J, Zhang K, Osman I and Bedogni B: MT1-MMP modulates melanoma cell dissemination and metastasis through activation of MMP2 and RAC1. Pigment Cell Melanoma Res 27: 287-296, 2014.

15. Tanaka M, Sato H, Takino T, Iwata K, Inoue M and Seiki M: Isolation of a mouse MT2-MMP gene from a lung cDNA library and identification of its product. FEBS Lett 402: 219-222, 1997.

16. Fillmore HL, VanMeter TE and Broaddus WC: Membrane-type matrix metalloproteinases (MT-MMPs): expression and function during glioma invasion. J Neurooncol 53: 187-202, 2001.

17. Davidson B, Goldberg I, Berner A, et al: Expression of membrane-type 1,2, and 3 matrix metalloproteinases messenger RNA in ovarian carcinoma cells in serous effusions. Am J Clin Pathol 115: 517-524, 2001

18. Docampo MJ, Cabrera J, Rabanal RM and Bassols A: Expression of matrix metalloproteinase-2 and -9 and membrane-type 1 matrix metalloproteinase in melanocytic tumors of dogs and canine melanoma cell lines. Am J Vet Res 72: 1087-1096, 2011.

19. Chen L, Di D, Luo G, et al: Immunochemical staining of MT2-MMP correlates positively to angiogenesis of human esophageal cancer. Anticancer Res 30: 4363-4368, 2010.

20. Ito E, Yana I, Fujita C, et al: The role of MT2-MMP in cancer progression. Biochem Biophys Res Commun 393: 222-227, 2010.

21. Abraham R, Schafer J, Rothe M, Bange J, Knyazev P and Ullrich A: Identification of MMP-15 as an anti-apoptotic factor in cancer cells. J Biol Chem 280: 34123-34132, 2005.

22. Zhu S, Zhou Y, Wang L, et al: Transcriptional upregulation of MT2-MMP in response to hypoxia is promoted by HIF-1 $\alpha$ in cancer cells. Mol Carcinog 50: 770-780, 2011.

23. Sobin LH and Fleming ID: TNM Classification of Malignant Tumors, fifth edition (1997). Union Internationale Contre le Cancer and the American Joint Committee on Cancer. Cancer 80: 1803-1804, 1997

24. Elias JM, Margiotta M and Gaborc D: Sensitivity and detection efficiency of the peroxidase antiperoxidase (PAP), avidin-biotin peroxidase complex (ABC), and peroxidase-labeled avidin-biotin (LAB) methods. Am J Clin Pathol 92: 62-67, 1989.

25. Ikeda N, Nakajima Y, Tokuhara $\mathrm{T}$, et al: Clinical significance of aminopeptidase N/CD13 expression in human pancreatic carcinoma. Clin Cancer Res 9: 1503-1508, 2003.

26. Hu Y, Liu J and Huang H: Recent agents targeting HIF-1 $\alpha$ for cancer therapy. J Cell Biochem 114: 498-509, 2013.

27. Chen $\mathrm{C}$ and $\mathrm{Yu} \mathrm{Z}$ : siRNA targeting HIF-1alpha induces apoptosis of pancreatic cancer cells through NF-kappaB-independent and -dependent pathways under hypoxic conditions. Anticancer Res 29: 1367-1372, 2009 .

28. Plaisier M, Koolwijk P, Hanemaaijer R, et al: Membrane-type matrix metalloproteinases and vascularization in human endometrium during the menstrual cycle. Mol Hum Reprod 12: 11-18, 2006.

29. Quick RE, Dunlap JA and Jessen JR: Expression analysis of zebrafish membrane type-2 matrix metalloproteinases during embryonic development. Gene Expr Patterns 12: 254-260, 2012.

30. Yamahatsu K, Matsuda Y, Ishiwata T, Uchida E and Naito Z: Nestin as a novel therapeutic target for pancreatic cancer via tumor angiogenesis. Int J Oncol 40: 1345-1357, 2012.

31. Nanashima A, Shibata K, Nakayama T, et al: Relationship between microvessel count and clinicopathological characteristics and postoperative survival in patients with pancreatic carcinoma. Hepatogastroenterology 59: 1964-1969, 2012.

32. Iki K, Tsutsumi M, Kido A, et al: Expression of matrix metalloproteinase 2 (MMP-2), membrane-type $1 \mathrm{MMP}$ and tissue inhibitor of metalloproteinase 2 and activation of proMMP-2 in pancreatic duct adenocarcinomas in hamsters treated with $\mathrm{N}$-nitrosobis(2-oxopropyl)amine. Carcinogenesis 20: 1323-1329, 1999. 